Please, find below the TEX file for the text, the TEX file for the Tables, the TEX file for the first figures and the POSTCRIPT file for the third figure.

\author{
$* * * * * * * * * * * * * * * * * * * * * * * * * * * * * * * * * * * * * * * * * * * * * * * * * * * * * * * * *$ \\ May 1994

\section{PATTERNS OF QUARK MASS MATRICES IN A CLASS OF CALABI-YAU MODELS}

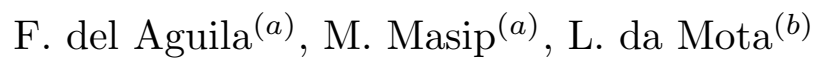 \\ (a) Departamento de Física Teórica y del Cosmos \\ Universidad de Granada \\ 18071 Granada, Spain \\ (b) Department of Theoretical Physics \\ University of Oxford \\ 1 Keble Road, Oxford OX1 3NP, UK
}

UG-FT-38/94

OUTP-94-6P

\begin{abstract}
We study a class of superstring models compactified in the 3-generation Calabi-Yau manifold of Tian and Yau. Our analysis includes the complete $E_{6}$-singlet sector, which has been recently evaluated using techniques of spectral and exact sequences. We use the discrete symmetries of the models to find flat directions of symmetry breaking that leave unbroken a low energy matter parity and make all leptoquarks heavy while preserving light Higgs fields. Then we classify the patterns of ordinary quark mass matrices and show that (without invoking effects due to nonrenormalizable terms) only one structure can accommodate the observed value of fermion masses and mixing angles, with preference for a heavy top quark $\left(m_{t} \geq 170 \mathrm{GeV}\right.$ for $\left.V_{13} \leq 0.013\right)$. The model, which unifies perturbatively and predicts a realistic structure of quark mass matrices with texture zeroes, is one of the many possible string vacua. However, in contrast with what is often assumed in the search for realistic unified scenarios, it is highly nonminimal near the unification scale and the predicted mass matrices have no simple symmetry properties.
\end{abstract}




\section{INTRODUCTION}

From the appearance of grand unified theories, the idea of unification of gauge couplings and the possibility of understanding the assignment of standard quantum numbers, the replication of families, and the pattern of fermion masses and mixing angles have been generic requirements in the search for a fundamental theory at very large energy scales [1]. The stability of the different mass scales involved (the electroweak and the unification scales) seems to demand a supersymmetric model. In addition, the proximity of the unification and the Planck scales points to the necessity of including gravity. String theory [2] has the potential of realizing all these ideas consistently. Hence, a program for obtaining the standard model from the string was proposed [3]. However, it is not known how to derive the physical vacuum from the string. Alternatively, one can scan compactified string models requiring consistency with the observed phenomenology. Possible candidates are the models resulting from the ten dimensional $E_{8} \times E_{8}$ heterotic superstring compactified on the 3-generation Calabi-Yau manifold of Tian and Yau [4]. In this paper we analyze the class of Tian-Yau models with a gauge symmetry

$$
G=S U(3)_{C} \times S U(3)_{L} \times S U(3)_{R} \subset E_{6}
$$

and a group of discrete symmetries of order 72 [5] (see Section 1.1). We use the available techniques to study this class of models quantitatively in some detail and, as a result, we single out only one realistic case. Our approach goes updown from the compactification to the electroweak scale. It is remarkable that, in contrast with the phenomenological approach going down-up, the model found is highly nonminimal near the unification scale. Hence, this is an example of a consistent model derived from the string which does not share the simplicity and predictivity of the minimal supersymmetric grand unified scenarios [6,7]. The perturbative unification takes place with a nonminimal matter content and after several intermediate scales of symmetry breaking. Moreover, the fermion mass matrices, although with a perturbative structure and texture zeroes, are not simple (symmetric). 
The phenomenological aspects of this class of models have been discussed previously, and the possibility of obtaining an almost minimal supersymmetric standard model at low energy and a realistic pattern of fermion masses and mixings emphasized $[5,8]$. This analysis made use of unknown soft supersymmetry breaking terms, nonrenormalizable terms, and $E_{6}$-singlet fields. Recently the $E_{6}$-singlet sector has been calculated using cohomology techniques of spectral sequences [9]. Here we reanalyse the low energy predictions of these models when the complete singlet and nonsinglet spectra and their renormalizable couplings at the compactification scale are included. We will assume that supersymmetry breaking [10] occurs and generates the required soft breaking terms. Nonrenormalizable terms [11] have not been worked out. We assume that they are largely suppressed and, in consequence, do not spoil the flatness conditions and introduce at most order $1 \mathrm{TeV}$ mass contributions for the different fields. We search for realistic models requiring that the intermediate scales of symmetry breaking (see next section)

(i) preserve a low-energy matter parity,

(ii) give heavy masses to all the leptoquarks,

(iii) keep the standard Higgs fields light, and

(iv) take place along flat directions of the potential.

Then we work out the low-energy spectrum and the ordinary fermion mass matrix predictions. We come out with 4 types of structures, but only one can accommodate the observed quark masses and the Cabibbo-Kobayashi-Maskawa matrix $V_{i j}$. Ramond, Roberts, and Ross (RRR) [7] have stitched the Yukawa quilt classifying the possible patterns of symmetric (or hermitian) quark mass matrices with texture zeroes. These matrices incorporate the perturbative structure observed in the quark sector: a heavy third family and the rest of masses expressed as an expanssion in powers of $V_{12}$. As they point out, these matrices are a natural expectation in compactified string theory with broken discrete symmetries. The four types $(A$, $B, C, D)$ of patterns that we find are explicit realizations of their mechanism. We do not find, however, any of the RRR textures. Our matrices are not necessarily 
symmetric and contain less texture zeroes.

In what follows we present the class of models, the two $Z_{N}$ matter parities, $P_{2,3}$, which can be defined in this class, and illustrate the type of perturbative structures and texture zeroes which give nontrivial constraints on fermion masses and mixing angles. In Section II (III) we analyse the models with $P_{3}\left(P_{2}\right)$ matter parity. We argue that only the model with mass matrices of type $D$ is phenomenologically viable and discuss the flatness conditions for this model is some detail. Section IV is devoted to conclusions.

\subsection{THE CLASS OF MODELS}

The Tian-Yau manifold under study $[5,8,9]$ is constructed as the quotient manifold $R / G$, where $R$ is the space of solutions in $C P_{x}^{3} \times C P_{y}^{3}$ to

$$
\begin{gathered}
f(x)=\sum_{i=1}^{4}\left(x^{i}\right)^{3}=0 ; g(y)=\sum_{i=1}^{4}\left(y^{i}\right)^{3}=0 ; \\
h(x, y)=x^{1} y^{1}+x^{2} y^{2}+c\left(x^{3} y^{3}+x^{4} y^{4}\right)=0,
\end{gathered}
$$

and $G$ is the freely-acting $Z_{3}$ discrete group

$$
g:\left(x^{1}, x^{2}, x^{3}, x^{4} ; y^{1}, y^{2}, y^{3}, y^{4}\right) \rightarrow\left(x^{1}, \alpha^{2} x^{2}, \alpha x^{3}, \alpha x^{4} ; y^{1}, \alpha y^{2}, \alpha^{2} y^{3}, \alpha^{2} y^{4}\right) .
$$

This manifold defines a variety of unified models of electroweak and strong interactions with 3 chiral generations. Our choice of the complex structure (which is given in Eq. (2) in terms of the arbitrary parameter $c$ ) implies an order 72 group of discrete symmetries $[5,8]$. The nonsinglet matter content at the compactification scale $M_{c}$ consists of nine families of leptons $\lambda$, six of $\bar{\lambda}$, seven of quarks $q$ and antiquarks $Q$, and four of $\bar{q}$ and $\bar{Q}$, where

$$
\mathbf{2 7} \longrightarrow(\mathbf{1}, \overline{\mathbf{3}}, \mathbf{3}) \equiv \lambda+(\mathbf{3}, \mathbf{3}, \mathbf{1}) \equiv q+(\overline{\mathbf{3}}, \mathbf{1}, \overline{\mathbf{3}}) \equiv Q
$$

and the assignment of standard quantum numbers is

$$
\lambda \sim\left(\begin{array}{ccc}
h^{0} & h^{\prime-} & e \\
h^{+} & h^{\prime 0} & \nu \\
e^{c} & \nu^{c} & N
\end{array}\right) ; q \sim\left(\begin{array}{cc}
u & \\
d & \times 3 \text { colors } \\
d^{\prime} &
\end{array}\right) ; Q \sim\left(\begin{array}{cc}
u^{c} & \\
d^{c} & \times 3 \text { colors } \\
d^{\prime c} &
\end{array}\right) .
$$


The transformation properties of these fields under the group of discrete symmetries are listed in Table I.

The $E_{6}$-singlet sector of the Tian-Yau construction has been recently calculated by Hubsch and collaborators using the cohomology techniques of exact and spectral sequences [9]. These methods generalize previous results obtained via polinomial deformations, providing a parametrization of all the $\mathbf{2 7}, \overline{\mathbf{2 7}}$, and $\mathbf{1}$ fields and a framework to analyze their couplings. The models with the complex structure that we have chosen contain 20 singlets (this number jumps for special choices of the manifold, like in the R-symmetric case considered in Ref.[9]). Their tensor representatives and transformation properties are listed in Table II (the fields in Tables I,II correspond to $B$ eigenstates; we can also choose a basis of $C$ eigenstates, as in Table III). The masses that these fields receive through instanton corrections [11] may be consistently small, and, at any rate, their $\mathbf{1} \mathbf{2 7} \overline{\mathbf{2 7}}$ couplings with the nonsinglet sector make them an essential part of the model at $M_{c}$. We should remark that among the singlets calculated in Ref. [9] there is not a subset with transformation properties identical to those of $\lambda$ and $\bar{\lambda}$ fields. (In the past it was usually assumed, based on theorems on the dimension of various cohomology groups on the manifold, a one to one correspondence between $\mathbf{2 7}^{\prime} s$ and some $E_{6}$ singlets.)

Once the supersymmetry breaking terms are included, the effective theory is specified if we know the dominant terms in the superpotential. The trilinear terms must be invariant under the discrete symmetries of the model, and at first order in $\sigma$ perturbation theory can be calculated in terms of the parameter $c$ specifying the complex structure of the manifold $[8,9]$, up to normalization factors of the fields. We write in Tables IV and $\mathrm{V}$ all the nonzero trilinears of type $\lambda^{3}, \lambda q Q$ and $s \lambda \bar{\lambda}, s^{3}$, respectively. These are the relevant terms in the discussion of fermion masses and flat directions. Due to our lack of knowledge of the field normalization factors, the explicit expression of all the coefficients is not necessary. The symmetries of the manifold also restrict the possible nonrenormalizable terms, which 
appear nonperturbatively after integrating out the massive string and KaluzaKlein modes. In the present work we will use large singlet VEVs to make massive many of the vectorlike families that appear at $M_{c}$. This enables us to consistently neglect all nonrenormalizable terms. (The suppression could be justified by their nonperturbative origin.) The motivation to do this is phenomenological, since it has been shown that nonrenormalizable terms tend to give too large masses to the standard Higgses [12] and to spoil the flatness conditions [13]. Both problems could be avoided by the action of an exact discrete symmetry $[13,14]$, but in these models unbroken symmetries imply the presence of extra light fields giving an unacceptable value for the proton lifetime and/or low energy gauge couplings [15].

The rank-6 models under discussion require two large intermediate scales of gauge symmetry breaking defined by VEVs along $N$ and $\nu^{c}$ (plus identical $\bar{N}$ and $\bar{\nu}^{c} \mathrm{VEVs}$ ) in two different $\lambda+\bar{\lambda}$ multiplets. The first scale leaves a symmetry $S U(3)_{C} \times S U(2)_{L} \times S U(2)_{R} \times U(1)_{B-L}$, which is subsequently broken to the standard $S U(3)_{C} \times S U(2)_{L} \times U(1)_{Y}$ gauge group. Below the intermediate scales the exotic flavors in the three chiral 27s of $E_{6}$ (two down type quarks, two neutrinos, and two lepton/Higgs doublets, see Eq.(3)) will combine into nonchiral representations of the standard model gauge group and will become very massive.

\subsection{MATTER PARITY MODELS}

To guarantee the absence of the lowest dimension baryon and lepton number violating operators and then of a fast proton decay, an effective matter parity must be at work. It has been shown that only two $Z_{N}$ matter parities can be implemented in the class of models under study [16]. They correspond to $P_{2}=C g_{2}$, generating a $Z_{2}$ discrete group, and $P_{3}=B g_{3}$, which defines a $Z_{3}$ symmetry (see Table I for the definition of $B$ and $C$ ). The action of the gauge discrete symmetries $g_{2,3}$ on the $\mathbf{2 7}$ representation of $E_{6}$ and the transformation properties of the standard matter fields under $P_{2,3}$ are listed in Tables VI and VII, respectively. 
Both of these matter parities forbid in the low energy superpotential the terms

$$
l h, l l e^{c}, q l d^{c}, u^{c} d^{c} d^{c}
$$

( $l, h$, and $q$ stand for lepton, Higgs and quark doublets) while allowing for the standard Yukawa terms. As $P_{2,3}$ are low energy symmetries, the VEVs breaking the gauge group $G$ down to $S U(3)_{C} \times S U(2)_{L} \times U(1)_{Y}$ at large intermediate scales must lie along matter parity neutral directions. This implies that the $E_{6}$ singlets $s$ and the $S U(5)$ singlets $\nu^{c}$ and $N$ acquiring VEVs must live in the subspaces generated by the families in Table VIII.

\subsection{PATTERNS OF FERMION MASSES WITH TEXTURE ZEROES}

The intermediate scales will break the discrete symmetries of the model except for a low energy matter parity. Broken discrete symmetries, however, may define texture zeroes in the fermion mass matrices [7]. Moreover, the existence of different scales of symmetry breaking may generate the observed hierarchy of masses and mixing angles. To illustrate this, consider a $Z_{N} \times Z_{2}$ symmetry acting on three chiral families of fermions $f_{i}$ as

$$
\begin{aligned}
& \left(f_{1}, f_{2}, f_{3}\right) \rightarrow\left(\alpha^{-2} f_{1}, \alpha f_{2}, f_{3}\right), \\
& \left(f_{1}, f_{2}, f_{3}\right) \rightarrow\left(f_{1}^{c}, f_{2}^{c}, f_{3}^{c}\right),
\end{aligned}
$$

$\left(\alpha^{N}=1\right.$, with $\left.N \neq 2,3,4,6\right)$ with analogous transformation properties for the antifermions $f_{i}^{c}$. Now suppose that the discrete symmetry is broken in the Higgs sector, in such a way that the Higgs $h=\kappa_{1} h_{1}+\kappa_{2} h_{2}+\kappa_{3} h_{3}$ contains components in three families which transform under $Z_{N}$

$$
\left(h_{1}, h_{2}, h_{3}\right) \rightarrow\left(h_{1}, \alpha^{-2} h_{2}, \alpha h_{3}\right),
$$

and are neutral respect to the $Z_{2}$ symmetry. When $h$ developes a VEV, the structure of the corresponding fermion mass matrix will be

$$
f_{i}^{c}\left(\begin{array}{ccc} 
& f_{i} & \\
\cdot & C & \cdot \\
C & B & \cdot \\
\cdot & \cdot & A
\end{array}\right),
$$


where $A, B$, and $C$ are proportional to $\kappa_{1}, \kappa_{2}$, and $\kappa_{3}$, respectively. Moreover, since the components $\kappa_{i}$ may be proportional to ratios between intermediate scales (and/or $M_{c}$ ), the hierarchy of these coefficients could explain the observed pattern of fermion masses and mixings. The texture above, for instance, in the down quark mass matrix, could fit $m_{b}, m_{s}, m_{d}$ and predict (for a diagonal up-charm submatrix) $V_{12} \approx \sqrt{\frac{m_{d}}{m_{s}}} \approx 0.22$. Analogous mass matrix structures are found in the class of models under study.

\section{MODELS WITH $P_{3}$ MATTER PARITY}

In this section we classify the models with $P_{3}$ matter parity. We find that only three patterns of quark mass matrices are consistent with the conditions ( $i$ $i v$ ) discussed in section I. None of them, however, can accommodate the observed values of quark masses and mixings.

To preserve $P_{3}$ (condition (i)) the fields developing large VEVs must be combinations of the fields in Table VIII. The 4 vectorlike families of quarks $(u d)+\overline{(u d)}$ and $u^{c}+\overline{u^{c}}$ will get masses (condition (ii)) via renormalizable interactions only if one of the singlets $s_{2}, s_{6}, s_{14}$ and another of $s_{4}, s_{8}, s_{16}$ acquire VEVs. To avoid fast proton decay it is also necessary that the down type quarks $d^{\prime}$ in $q_{3}, q_{5}$ and $q_{7}$ become very massive. This only happens if $N$ in $\lambda_{2}, \lambda_{4}$ or $\lambda_{5}$ aquire $\operatorname{VEVs}(\langle N\rangle$ in $\lambda_{6}$ and $\lambda_{7}$ leave $d^{\prime}$ in $q_{3}$ massless). A remarkable fact in $P_{3}$ models is that the matter parity imposes the three chiral families of $(u d)$ and $u^{c}$ to be in $q_{3}, q_{5}, q_{7}$ and $Q_{3}, Q_{5}, Q_{7}$, respectively. This implies that below the intermediate scales the symmetries $A, D \times V_{d}$, and $P \times V_{d}$ in Table I remain unbroken in the up quark sector and may generate matrix textures à la Ramond-Roberts-Ross, as explained in the Introduction. These structures would be approximate in the down sector.

According to the $P_{3}$ assignments (note that the matter parity is an extra quantum number that distinguishes lepton from Higgs doublets) the standard model Higgses $h, h^{\prime}$ result from the diagonalization of the $9 \times 9$ matrix with $h$ in $\lambda_{2}, \lambda_{4}, \lambda_{5}, \lambda_{6}, \lambda_{7} ; \bar{h}^{\prime}$ in $\bar{\lambda}_{1}, \bar{\lambda}_{2}$; and $\bar{l}$ in $\bar{\lambda}_{5}, \bar{\lambda}_{6}$ (rows), and $h^{\prime}$ in $\lambda_{2}, \lambda_{4}, \lambda_{5}$, $\lambda_{6}, \lambda_{7} ; \bar{h}$ in $\bar{\lambda}_{1}, \bar{\lambda}_{2}$; and $l$ in $\lambda_{1}, \lambda_{8}$ (columns). For $\langle N\rangle$ in $\lambda_{2}$ and $\left\langle\nu^{c}\right\rangle$ in $\lambda_{3}$ 
or $\lambda_{9}$ we obtain a light Higgs pair (i.e., a rank 8 Higgs mass matrix) for singlet VEVs in $s_{2}, s_{4}$ or $s_{2}, s_{8}$ (condition (iii)). In these cases the light Higgs $h$ lies in $\lambda_{6}$ (without mixing with other families) and would predict patterns of up quark mass matrices antisymmetric (type $A$ ) to be discussed below. For singlet VEVs in $s_{2}, s_{8} ; s_{4}, s_{6}$; or $s_{6}, s_{8}$ there are two light Higgses after the intermediate scales (one of them along $\lambda_{6}$ ). These cases, however, are not consistent with the required flatness in the scalar potential (condition (iv)). For singlet VEVs in $s_{2}, s_{8}$, for instance, it is impossible to adjust to zero simultaneously the $N$ and $\nu^{c} D$-terms and the $F$-terms $F_{N_{4}}, F_{\nu_{3}^{c}}, F_{s_{2}}, F_{s_{4}}, F_{s_{6}}, F_{s_{14}}$, and $F_{s_{16}}$. The cases with $\langle N\rangle$ along $\lambda_{4}$ are analogous.

For $\langle N\rangle$ in $\lambda_{5}$ and $\left\langle\nu^{c}\right\rangle$ in $\lambda_{3}$ we obtain light Higgses $h, h^{\prime}$ for singlet VEVs in $s_{2}, s_{4}$; or $s_{2}, s_{8}$. The second case, however, does not take place along a flat direction and is excluded. The first case does define a flat direction, and gives the pattern of quark masses and mixings of type $B$ (see below). Finally, there are the models with $\langle N\rangle$ in $\lambda_{5}$ and $\left\langle\nu^{c}\right\rangle$ in $\lambda_{9}$. The case with singlet VEVs along $s_{4}, s_{6}$ does not lie along a flat direction, while the model with VEVs along $s_{2}, s_{4}$ gives the pattern of type $C$ discussed below.

\subsection{QUARK MASS MATRICES IN $P_{3}$ MATTER PARITY MODELS}

- Type A: In this case the up quark mass matrices are generically antisymmetric as a consequence of an exact symmetry. The $\overline{(u d)_{a}} M_{a b}(u d)_{b}$ and $\overline{u^{c}}{ }_{a} M_{a b} u_{b}^{c}$ mass matrices defining the three chiral families are identical, and then the states which correspond to the standard $(u d)$ and $u^{c}$ quarks lie in the same directions in flavor space. Therefore, as under $P \times V_{d}$ the Higgs $h\left(\in \lambda_{6}\right)$ is odd whereas $u(\in q)$ and $u^{c}(\in Q)$ transform into each other (see Table I), all contributions to the up quark mass matrices are antisymmetric. This implies $m_{t}=m_{c}$ and $m_{u}=0$, making all these cases unrealistic. 
- Type B: The quark mass matrices in this case are

$$
u_{j}^{c}\left(\begin{array}{ccc}
u_{i} & \\
\cdot & B & B^{\prime} \\
B & A^{\prime} & \cdot \\
B^{\prime} & \cdot & A
\end{array}\right), \quad d_{j}^{c}\left(\begin{array}{ccc}
D & \cdot & E \\
F & \cdot & G \\
\cdot & C & \cdot
\end{array}\right),
$$

with $A / A^{\prime}=B / B^{\prime}$. This structure can be disregarded only after a detailed analysis. It is possible to fit the six quark masses, with a heavy top quark, but the predicted mixings of the third family are far too small. In particular, we obtain $V_{23}<m_{c}^{2} / m_{t}^{2} \approx 10^{-4}$, while experimentally this mixing is two orders of magnitude larger $\left(V_{23} \approx 0.042 \pm 0.12[17]\right)$. The relatively large entries with a nonperturbative origin required to cure this problem would be in contradiction with our assumptions (see a more detailed discussion below).

- Type $C$ : This is the most interesting $P_{3}$ case. The quark mass matrices read

$$
u_{j}^{c}\left(\begin{array}{ccc}
\cdot & B & B^{\prime} \\
B & A^{\prime} & \cdot \\
B^{\prime} & \cdot & A
\end{array}\right), \quad d_{j}^{c}\left(\begin{array}{ccc}
C & \cdot & \cdot \\
\cdot & D & E \\
\cdot & F & G
\end{array}\right),
$$

with $A / A^{\prime}=B / B^{\prime}$ but otherwise arbitrary complex entries (since they depend on unknown normalization factors of the fields). The zeroes in these matrices are a remnant of the discrete symmetry $A$ in Table I. For example, for the up quarks we have:

$$
\left(u_{3}, u_{5}, u_{7}\right) \rightarrow\left(u_{3}, \alpha u_{5}, \alpha u_{7}\right)
$$

and for the Higgs $\left(h=\kappa_{1} h_{2}+\kappa_{2} h_{4}\right)$ :

$$
\left(h_{2}, h_{4}\right) \rightarrow\left(\alpha^{2} h_{2}, \alpha h_{4}\right)
$$

The up quark matrix is symmetric due to the $P \times V_{d}$ symmetry, whereas the relation between the coefficients follows from the $D$ symmetry:

$$
\left(u_{3}, u_{5}, u_{7}, h_{2}, h_{4}\right) \rightarrow\left(u_{3}^{c}, u_{7}^{c}, u_{5}^{c}, h_{4}, h_{2}\right)
$$


Although these structures are sensitive to top radiative corrections, to decide on the main features of these matrices the following approximate analysis will show up good enough. To avoid degeneracy on quark masses, $A \approx m_{t}$ and $A^{\prime} \approx m_{c}$ ( $A^{\prime} \approx m_{t}$ and $A \approx m_{c}$ give equivalent results). On the other hand, since the first column of the down quark matrix does not mix with the other two, $B$ in the up quark matrix is fixed by the Cabbibo angle $\left(\approx V_{12}\right): B \approx A^{\prime} V_{12} \approx m_{c} V_{12}$, implying $B^{\prime} \approx \frac{m_{c}^{2}}{m_{t}}$. In the down quark matrix $C \approx m_{d}$ and the other nonzero entries give $m_{s}, m_{b}$, and $V_{23}$. The mixing $V_{23}$ comes mainly from the down sector because $B^{\prime} \approx 0$, which also implies $V_{13} \approx V_{12} V_{23}$ and $m_{u} \approx m_{c} V_{12}^{2}$. The last relation gives a too high estimate of $m_{u} \approx 40 \mathrm{MeV}$.

The value of $m_{u}$ that we obtain seems the only bad prediction of type $C$ matrices, and we wonder if contributions from nonrenormalizable terms could change it. In order to fulfill conditions (iii) and (iv), throughout the paper we have assumed a generic suppression by 13 orders of magnitude for these terms and based our analysis on trilinear (renormalizable) couplings. To predict an acceptable value of $m_{u}$ higher order contributions should introduce a direct up mass term or a mixing term between the first and second down quark families. These contributions would be of order $V_{12}^{2} m_{c} / m_{t} \approx 10^{-5}$ times the Yukawa contribution corresponding to the top quark mass, which seems to be much higher than the suppression required by conditions (iii,iv). Similar but even more severe comments apply to pattern $B$ above. There the bad prediction is $V_{23}<10^{-4}$, and to fix it one should rely on nonrenormalizable contributions with a suppression of order $10^{-2}$ relative to trilinear entries and still keep the Higgs fields light and the flatness conditions.

\section{MODELS WITH $P_{2}$ MATTER PARITY}

An analogous exploration can be done for models with the $Z_{2}$ matter parity $P_{2}$. It is convenient to write the superpotential in terms of $C$ eigenstates (see Table III), which we denote with primes. In $P_{2}$ models the Higgs $h, h^{\prime}$ result from the diagonalization of a $11 \times 11$ mass matrix where the rows correspond to $h$ in $\lambda_{1}^{\prime}, \lambda_{3}^{\prime}, \lambda_{5}^{\prime}, \lambda_{6}^{\prime}, \lambda_{8}^{\prime} ; \bar{h}^{\prime}$ in $\bar{\lambda}_{1}^{\prime}, \bar{\lambda}_{2}^{\prime}$; and $\bar{l}$ in $\bar{\lambda}_{3}^{\prime}, \bar{\lambda}_{4}^{\prime}, \bar{\lambda}_{5}^{\prime}, \bar{\lambda}_{6}^{\prime}$, and the columns to $h^{\prime}$ in 
$\lambda_{1}^{\prime}, \lambda_{3}^{\prime}, \lambda_{5}^{\prime}, \lambda_{6}^{\prime}, \lambda_{8}^{\prime} ; \bar{h}$ in $\bar{\lambda}_{1}^{\prime}, \bar{\lambda}_{2}^{\prime}$, and $l$ in $\lambda_{2}^{\prime}, \lambda_{4}^{\prime}, \lambda_{7}^{\prime}, \lambda_{9}^{\prime}$. In $P_{2}$ models the three chiral families of quarks will be combinations of the families $q_{1}^{\prime}, q_{2}^{\prime}, q_{3}^{\prime}, q_{4}^{\prime}$, and $q_{6}^{\prime}$, with all discrete symmetries (except for $P_{2}$ ) broken in the quark sector. Then, it is not obvious, but there are restricted patterns of quark mass matrices in these models.

For singlet VEVs along $s_{1}^{\prime}, s_{3}^{\prime}, s_{13}^{\prime}, s_{15}^{\prime}$ we obtain models which satisfy simultaneously the flatness conditions while making all vectorlike quarks heavy. In all these models (for any choice of $N$ and $\nu^{c} \mathrm{VEVs}$ ), however, the Higgs $h$ which couples to up quarks lies in $\lambda_{6}^{\prime}$ and/or in pure $\bar{\lambda}^{\prime}$ fields. The first case gives antisymmetric matrices (pattern type $A$ discussed above), and the second has no Yukawa couplings and implies massless up quarks. Any other combination of VEVs, except for two equivalent choices, do not satisfy conditions (ii) and (iv) simultaneously. The only interesting case is obtained when the singlet VEV has components along $s_{1}^{\prime}, s_{7}^{\prime}, s_{13}^{\prime}$, whereas $\langle N\rangle$ is along $\lambda_{1}^{\prime},\langle\bar{N}\rangle$ along $\bar{\lambda}_{1,2}^{\prime},\left\langle\nu^{c}\right\rangle$ along $\lambda_{7}^{\prime}$ and $\left\langle\bar{\nu}^{c}\right\rangle$ along $\bar{\lambda}_{3,4}^{\prime}$. (The equivalent choice is obtained by a $D \times V_{d}$ transformation.)

Let us concentrate on this case. From now on we drop the primes for specifying $C$ eigenstates.

\subsection{FLATNESS CONDITIONS}

For the vacuum under consideration $D$-flatness implies

$$
\begin{aligned}
\left\langle\nu_{7}^{c}\right\rangle & =\left\langle{\overline{\nu^{c}}}_{3}+\bar{\nu}_{4}\right\rangle, \\
\left\langle N_{1}\right\rangle & =\left\langle\bar{N}_{1}+\bar{N}_{2}\right\rangle,
\end{aligned}
$$

while $F$-flatness imposes

$$
\begin{aligned}
\left\langle F_{\nu_{4}^{c}}\right\rangle & =\left\langle c_{3} s_{7} \bar{\nu}_{3}^{c}+c_{4} s_{7} \bar{\nu}_{4}^{c}\right\rangle=0, \\
\left\langle F_{N_{3}}\right\rangle & =\left\langle c_{1} s_{1} \bar{N}_{1}+c_{2} s_{1} \bar{N}_{2}+c_{9} s_{13} \bar{N}_{1}+c_{10} s_{13} \bar{N}_{2}\right\rangle=0, \\
\left\langle F_{s_{3}}\right\rangle & =\left\langle c_{1} N_{1} \bar{N}_{1}-c_{2} N_{1} \bar{N}_{2}+d_{7} s_{7}^{2}\right\rangle=0, \\
\left\langle F_{s_{15}}\right\rangle & =\left\langle c_{9} N_{1} \bar{N}_{1}-c_{10} N_{1} \bar{N}_{2}+d_{8} s_{7}^{2}\right\rangle=0, \\
\left\langle F_{s_{1}}\right\rangle & =\left\langle 3 d_{1} s_{1}^{2}+2 d_{2} s_{1} s_{13}+d_{4} s_{13}^{2}\right\rangle=0, \\
\left\langle F_{s_{13}}\right\rangle & =\left\langle d_{2} s_{1}^{2}+3 d_{3} s_{13}^{2}+2 d_{4} s_{1} s_{13}\right\rangle=0 .
\end{aligned}
$$


(The Yukawa couplings involving the gauge singlets were defined in Table V.) The rest of F-terms are trivially zero. The 8 equations have to be solved adjusting 9 VEVs. The 2 equations for $\left\langle\nu_{7}^{c}\right\rangle,\left\langle\bar{\nu}_{3}^{c}\right\rangle$ and $\left\langle\bar{\nu}_{4}^{c}\right\rangle$ decouple from the rest, defining a flat direction. The 4 homogeneous equations involving $\left\langle N_{1}, \bar{N}_{1,2}\right\rangle$ and $\left\langle s_{7}\right\rangle$ can be solved as a function of $\left\langle\frac{s_{13}}{s_{1}}\right\rangle$ and $c$ but after modifying $\left\langle F_{s_{3}}\right\rangle$ and/or $\left\langle F_{s_{15}}\right\rangle$, for otherwise the corresponding equations are incompatible. This is so because (we follow the procedure of Ref. [9] to calculate the Yukawa couplings)

$$
\frac{c_{2}}{c_{1}}=\frac{c_{10}}{c_{9}}=\sqrt{\frac{3}{2}},
$$

whereas $d_{7}$ and $d_{8}$ have a different $c$ dependence. The simplest solution is to assume a large singlet mass term $M s_{1,13} s_{3,15}$, with $M \approx M_{c}$ if the VEVs have to be of the correct size. Although nonrenormalizable terms and singlet masses have both nonperturbative origin, due to the exponential behaviour of the scales generated nonperturbatively [11] nothing prevents a very different suppression for those two types of terms (as required here). Finally, $\left\langle F_{s_{1,13}}\right\rangle=0$ fix $\left\langle\frac{s_{13}}{s_{1}}\right\rangle$ and $c$ because they are homogeneous and compatible only for a definite choice of Yukawa couplings, and then of $c$. The prove that the preferred vacuum alignment is the one just discussed also requires to know the soft scalar masses that trigger the VEVs.

\subsection{QUARK MASS MATRICES IN THE $P_{2}$ MATTER PARITY MODEL}

Once fixed the pattern of symmetry breaking, we can study the spectrum of fields that remain light and, in particular, identify the three chiral families of quarks and leptons. In Figs. 1-2 we give the resulting mass matrices for lepton doublets $(1 a)$, charged lepton singlets $(1 b)$, Higgs doublets $(1 c)$, down-quark singlets $(2 a)$, and up-quark singlets and quark doublets which coincide $(2 b)$. We use $\left\langle N_{1}\right\rangle \sim\left\langle\bar{N}_{1,2}\right\rangle \sim x,\left\langle\nu_{7}^{c}\right\rangle \sim\left\langle\bar{\nu}_{3,4}^{c}\right\rangle \sim y,\left\langle s_{13}+s_{14}\right\rangle \sim r,\left\langle s_{7}\right\rangle \sim s$ to specify the order of magnitude of the entries. A dot stands for a zero due to the $P_{2}$ symmetry. Blanks may be eventually filled out with nonrenormalizable contributions of order $1 \mathrm{TeV}$. Gaugino mass generation has been taken into account introducing one pair 
of doublets in $(2 a)$ and two pairs of singlets in $(2 b)$. Diagonalizing these matrices we find the three chiral families of quarks and leptons:

$$
\begin{array}{cccc}
\left(\begin{array}{l}
u \\
d
\end{array}\right): & q_{3}, & \alpha_{1} q_{1}+\alpha_{2} q_{2}+\alpha_{3} q_{4}, & \beta_{1} q_{6}+\beta_{2} q_{2}+\beta_{3} q_{4} ; \\
u^{c}: & u^{c}{ }_{3}, & \alpha_{1} u^{c}{ }_{1}+\alpha_{2} u^{c}{ }_{2}+\alpha_{3} u^{c}{ }_{4}, & \beta_{1} u^{c}{ }_{6}+\beta_{2} u^{c}{ }_{2}+\beta_{3} u^{c}{ }_{4} ; \\
d^{c}: & d^{c}{ }_{3}, & \gamma_{1} d^{c}{ }_{1}+\gamma_{2} d^{c}{ }_{2}+\gamma_{3} d^{c}{ }_{4}+\gamma_{4} d^{c}{ }_{6}, & \delta_{1} d^{\prime c}{ }_{5}+\delta_{2} d^{c}{ }_{2}+\delta_{3} d^{c}{ }_{4}+\delta_{4} d^{c}{ }_{6} ; \\
& \\
& e^{c}: \quad e^{c}, & \epsilon_{1} l_{1}+\epsilon_{2} h_{7}^{\prime}+\epsilon_{3} h_{9}^{\prime}, & l_{6}, \quad l_{8} ; \\
& e_{6}^{c}, e^{c} .
\end{array}
$$

There are two pairs of Higgs doublets light after the intermediate scales (see Fig. 1c). These Higgses receive order $1 \mathrm{TeV}$ masses only from nonrenormalizable (and soft SUSY breaking) effects. We will assume that these masses mix them and that the pair not involved in the electroweak phase transition is massive enough $(\sim 1 \mathrm{TeV})$ to avoid flavour changing neutral currents via Yukawa interactions. Then the Higgs components along $\mathbf{2 7}$ families are

$$
\begin{array}{ll}
\left(\begin{array}{l}
h^{0} \\
h^{+}
\end{array}\right): & \kappa_{1} h_{6}+\kappa_{2} h_{1} ; \\
\left(\begin{array}{c}
h^{\prime-} \\
h^{\prime 0}
\end{array}\right): & \kappa_{1}^{\prime} h_{6}^{\prime}+\kappa_{2}^{\prime} h_{5}^{\prime}+\kappa_{3}^{\prime} h_{8}^{\prime}+\kappa_{4}^{\prime} h_{3}^{\prime}+\kappa_{5}^{\prime} l_{7} .
\end{array}
$$

All nonstandard lepton doublets and quarks get heavy masses at the intermediate scales. However, in addition to the extra pair of Higgs doublets, one pair $\left(e^{c}, \bar{e}^{c}\right)$ of charged lepton singlets remains light (see Fig. 1b), with masses of order $1 \mathrm{TeV}$ given by nonrenormalizable interactions.

When $h$ and $h^{\prime}$ develope $\operatorname{VEVs}\left(v\right.$ and $v^{\prime}$, respectively) ordinary quarks and leptons get masses through the Yukawa couplings in Table IV. To avoid a Higgs along $h_{6}$ only $\left(\kappa_{2}=0\right)$ and then an antisymmetric up quark matrix, $s$ must be of the same order as $x, y$. Now, at zero order in $r / s\left(\alpha_{2}=\alpha_{3}=0, \beta_{2}=\beta_{3}=0\right.$, $\gamma_{i}=\delta_{i}=0$ for $i \neq 1$ ) there are only two entries in the up quark matrix: $A \sim m_{t}$ and $B$, which would be much smaller than $A$ if $\kappa_{1} \ll \kappa_{2}$; and two entries in the down quark matrix: $A^{\prime} \sim m_{b}$ and $B^{\prime}$, verifying $B^{\prime} \ll A^{\prime}$ if $\kappa_{2}^{\prime} \ll \kappa_{3}^{\prime}$. At zero order 
in $\kappa_{2}^{\prime} / \kappa_{3}^{\prime}$, there are two entries in the charged lepton matrix: $A_{1}^{\prime \prime}$ and $A_{2}^{\prime \prime}$, which should satisfy $A_{1}^{\prime \prime 2}+A_{2}^{\prime \prime 2} \sim m_{\tau}^{2}$. In consequence, at zero order in $r / s, \kappa_{2} / \kappa_{1}$, and $\kappa_{2}^{\prime} / \kappa_{3}^{\prime}$ only the third family is massive. The structure in the fermion matrices will appear as a perturbation on these ratios. The complete mass matrices are

- Type D:

$u_{j}^{c}\left(\begin{array}{ccc}\cdot & D & E \\ D & \cdot & C+B \\ E & C-B & A\end{array}\right) ; \quad d_{j}^{c}\left(\begin{array}{ccc}B^{\prime} & \cdot & \cdot \\ \cdot & C^{\prime} & A^{\prime} \\ \cdot & D^{\prime} & E^{\prime}\end{array}\right) ; \quad e_{j}^{c}\left(\begin{array}{ccc}C^{\prime \prime} & B^{\prime \prime} & A_{2}^{\prime \prime} \\ \cdot & D^{\prime \prime} & \cdot \\ E^{\prime \prime} & \cdot & A_{1}^{\prime \prime}\end{array}\right)$,

where $A, A^{\prime}, A_{1}^{\prime \prime}$, and $A_{2}^{\prime \prime}$ are zero order entries and the rest correspond to higher order on the three ratios above. All entries are arbitrary complex coefficients, with the zeroes as a remnant of the $A \times B \times P \times V_{d}$ discrete symmetry in Table I. (Although $A$ and $B$ denote both discrete symmetries and mass matrix entries, no confusion is expected by the use of this notation.)

All quark masses are taken at $1 \mathrm{GeV} . m_{t}$, which we will define as $h_{t} / v$ at $M_{Z}$, must be evolved down to $1 \mathrm{GeV}$. The QCD running coefficient is a factor $\approx 1.8$. Since $A \sim m_{t}^{\prime} \equiv 1.8 m_{t}$ and in the down quark matrix the third family does not participate of the first column, $E \approx m_{t} V_{13}$ and $C^{2}-B^{2} \approx m_{c} m_{t}^{\prime}$. The masses $m_{d}$ and $m_{s}$ and the mixing $V_{23}$ fix $B^{\prime}, C^{\prime}$, and $D^{\prime}: B^{\prime} \approx m_{d}, C^{\prime} \approx V_{23} m_{b}$ and $D^{\prime} \approx m_{s}$; whereas for the Cabibbo mixing, $V_{12}$, one has $D \approx m_{c} V_{12}$. Then $m_{u}$ results from the cancellation

$$
m_{u} \approx m_{c} V_{12}^{2}-1.8 m_{t} V_{13}^{2} .
$$

This correlation translates into a preference for a large top quark mass and $V_{13}$ mixing. For example, if $V_{13} \leq 0.013$, the top quark mass must be $m_{t} \geq 170 \mathrm{GeV}$ (for lower values of $V_{13}$ it is necessary to include top radiative corrections and a more precise diagonalization of the matrices to give an estimate of the correlation). The three charged lepton masses can be easily adjusted using the arbitrariness in 
the third matrix. A detailed numerical analysis of the range of variation of the different (physical) parameters will be presented elsewhere.

\subsection{OTHER PHENOMENOLOGICAL IMPLICATIONS OF THE MODEL}

Other aspect of the model are the phenomenological implications of the nonstandard $\left(e^{c}, \bar{e}^{c}\right)$ pair of leptons in the TeV region. They are even under $P_{2}$, and then their only couplings in the superpotential are

$$
P=a l l e^{c}+b h^{\prime} h^{\prime} e^{c}+c h h \bar{e}^{c}+m e^{c} \bar{e}^{c} .
$$

For reasonable values of their masses and couplings their presence will not be in conflict with any particle physics experiment. However, we note that these trilinears explicitly break lepton number and could have relevance in Cosmology (baryogenesis).

Finally, we consider the perturbative unification of the gauge couplings in the model under study. The extra pair of Higgs doublets and charged lepton singlets, with masses around $1 \mathrm{TeV}$, provides an evolution of the running couplings which differs from the successful evolution suggested in the minimal supersymmetric scenario $\left(\alpha_{C}=\alpha_{L}=\alpha_{Y}\right.$ at $M_{X} \approx 10^{16} \mathrm{GeV}$, see Fig. 3). In this model, however, the presence of two intermediate scales and a large number of vectorlike families below the compactification scale seems to introduce enough arbitrariness to obtain the right value of the electroweak mixing angle. For $\sin _{W}^{2}=0.23$ the matter content implies that $\alpha_{L}=\alpha_{Y}$ at $M \approx 10^{15} \mathrm{GeV}$ (see Fig. 3), which sets the second intermediate scale $\left\langle\nu^{c}\right\rangle$. Above this scale our model has left-right symmetry (i.e., $\left.\alpha_{L}=\alpha_{R}\right)$. The unification of these couplings with $\alpha_{C}$ at $M_{c} \geq 10^{16} \mathrm{GeV}$ requires the presence of nonstandard quarks below $M_{c}$. Taking three down quark singlets $d^{c}+\overline{d^{c}}$ with masses around $10^{12} \mathrm{GeV}$ or 2 complete families of $q+\bar{q}, Q+\bar{Q}$, $d^{\prime}+d^{\prime c}$ at $10^{15} \mathrm{GeV}$ (the case plotted), and the rest of vectorlike families with a mass $M_{c}$, we obtain $\sin _{W}^{2}=0.23$ and a sensible unification scale $M_{c}$ (see Fig. 3).

\section{SUMMARY AND CONCLUSIONS}


We have discussed all the possible patterns of quark mass matrices in the first and most extensively studied class of 3-generation Calabi-Yau models. Our analysis is based on the discrete symmetries of the models, and it includes the complete matter content ( $E_{6}$-singlet and -nonsinglet fields) and their trilinear couplings at the compactification scale. To select the realistic cases, we have required an unbroken low-energy matter parity (a $Z_{2}$ or a $Z_{3}$ discrete symmetry), absence of light leptoquarks (which would be inconsistent with the proton lifetime and perturbative unification), and intermediate scales defining flat directions at the renormalizable level. The models with a $P_{3}$ matter parity left give 3 patterns of quark mass matrices; none of them, however, is able to accommodate a realistic spectrum of masses and mixing angles. Among the models with a $P_{2}$ matter parity we find only one case (studied in detail in sections 3.1-3.3) which seems free of inconsistencies, predicting an acceptable pattern of fermion masses and mixings, with preference for a heavy top quark $\left(m_{t} \geq 170 \mathrm{GeV}\right.$ for $\left.V_{13} \leq 0.013\right)$.

The model we have singled out can be derived from the string and is realistic. However, it can be seen as a counterexample of the present searches for realistic low-energy supersymmetric models. These are mainly based on two observations: that perturbative unification in the minimal model predicts the electroweak mixing angle with great accuracy and that the observed pattern of fermion masses and mixing angles can be explained with simple matrix structures (symmetric and with texture zeroes). The model found is nonminimal, has abundant extra matter near the unification scale, and has two large intermediate scales of symmetry breaking. In addition, although the fermion mass matrices have texture zeroes and a perturbative structure which is remnant of broken discrete symmetries, these are not symmetric nor simple.

\section{Acknowledgments}

We thank G.G. Ross for enlightening discussions.

\section{References}


[1] For a review on grand unification models, see G.G. Ross, Grand Unification Theories (Frontiers in Physics, Redwood City, CA, 1984).

[2] For a review on string theory, see M. Green, J. Schwarz and E. Witten, Superstring Theory (Cambridge University Press, Cambridge, 1987).

[3] P. Candelas, G. Horowitz, A Strominger, and E. Witten, Nucl. Phys. B258 (1985) 75.

[4] S.T. Yau, in Simposium on Anomalies, Geometry and Topology, ed. W.A. Bardeen and A.R. White (World Scientific, Singapore, 1985).

[5] B.R. Green, K.H. Kirklin, P. Miron and G.G. Ross, Nucl. Phys. B278 (1986) 667; Nucl. Phys. B292 (1987) 606; G.G. Ross, lectures given at 1988 Banf Summer Institute, CERN preprint TH-5109/88.

[6] S. Dimopoulos, L.J. Hall, and S. Raby, Phys. Rev. Lett. 68 (1992) 1984; J. Distler, Phys. Rev. D45 (1992) 4195; H. Arason, D.J. Castaño, P. Ramond, and E.J. Piard, Phys. Rev. D47 (1993) 1232.

[7] P. Ramond, R.G. Roberts, and G.G. Ross, Nucl. Phys. B406 (1993) 19; see also L. Ibáñez, and G.G. Ross, Oxford preprint OUTP-9403; FTUAM94/7.

[8] B.R. Green, K.H. Kirklin, P. Miron and G.G. Ross, Phys. Lett. B192 (1987) 111; J. Distler, B.R. Green, K.H. Kirklin, P. Miron and G.G. Ross, Phys. Lett. B195 (1987) 41.

[9] M.G. Eastwood and T. Hubsch, Commun. Math. Phys. 132 (1990) 383; P. Berglund, L. Parkes, and T. Hubsch, Commun. Math. Phys. 148 (1992) 57; P. Berglund, T. Hubsch, and L. Parkes, Mod. Phys. Lett. A5 (1990) 1485.

[10] H.P. Nilles, Phys. Lett. 115B (1982) 193; M. Dine, R. Rohm, N. Seiberg, and E. Witten, Phys. Lett. 156B (1985) 55.

[11] M. Dine, N. Seiberg, X. Wen, and E. Witten, Nucl. Phys. B278 (1986) 769; Nucl. Phys. B289 (1987) 319.

[12] F. del Aguila, G.D. Coughlan, and M. Masip, Phys. Lett. 227B (1989) 55.

[13] G. Lazarides, P.K. Mohapatra, C. Panagiotakopoulos and Q. Shafi, Nucl. Phys. B233 (1989) 614; Berglund and T. Hubsch, Phys. Lett. 260B (1991) 32. 
[14] G. Lazarides, C. Panagiotakopoulos, and Q. Shafi, Phys. Lett. 225B (1989) 66.

[15] F. del Aguila, G.D. Coughlan, and M. Masip, Nucl. Phys. B351 (1991) 90. [16] M.C. Bento, L. Hall and G.G. Ross, Nucl. Phys. B292 (1987) 400; G. Lazarides and Q. Shafi, Nucl. Phys. B338 (1990) 442.

[17] Review of Particle Properties, M. Aguilar Benítez et al., Phys. Rev. D45 (1992) 1. 


\section{Table captions}

Table I: Tensor representatives and transformation properties of the nonsinglet fields under the generators of the group of discrete symmetries. The transformations for the $Q$ and $\bar{Q}$ multiplets follow from interchanging $q \leftrightarrow Q$ and $\bar{q} \leftrightarrow \bar{Q}$. $\left(\alpha^{3}=1.\right)$

Table II: Tensor representatives and transformation properties of the $E_{6}$-singlet fields under the generators of the group of discrete symmetries.

Table III: $C$-eigenstates (primed) in terms of $B$-eigenstates. $B$ and $C$ are defined in Tables I,II.

Table IV: Trilinear couplings of type $\lambda^{3}$ and $\lambda q Q$ allowed by the discrete symmetries of the compactified model. We specify the terms in the $B$ and the $C$ bases.

Table V: Trilinear couplings of type $s \lambda \bar{\lambda}$ and $s^{3}$ (in the $C$ basis) allowed by the discrete symmetries.

Table VI: Transformation properties of the flavors in a $\mathbf{2 7}$ irrep of $E_{6}$ under the discrete gauge symmetries $g_{2}$ and $g_{3} \cdot\left(\alpha^{3}=1\right.$.)

Table VII: Transformation properties of the standard quark, lepton, and Higgs superfields under the matter parities $P_{2}$ and $P_{3}$.

Table VIII: Neutral flavors under the discrete symmetries $P_{2}$ and $P_{3}$. Large VEVs along these directions leave unbroken the corresponding low energy matter parity.

\section{Figure captions}

Figure 1: Mass matrices for lepton doublets (a), charged lepton singlets (b), and Higgs doublets (c).

Figure 2: Mass matrices for down quark singlets (a) and quark doublets and up quark singlets (b), which coincide.

Figure 3: Unification of running coupling constants for $\sin ^{2} \theta_{W}=0.23$ in the supersymmetric model with minimal matter content (dashes) and nonminimal supersymmetric model with an extra $h+h^{\prime}$ and $e^{c}+\bar{e}^{c}$ at $1 \mathrm{TeV}$ (solid). This 
model implies left-right unification at a scale $x \approx 10^{15} \mathrm{GeV}$. At this scale we add two families of $q+\bar{q}, Q+\bar{Q}$, and $d^{\prime}+d^{\prime c}$. 
This figure "fig1-1.png" is available in "png" format from: http://arxiv.org/ps/hep-ph/9406364v1 
This figure "fig2-1.png" is available in "png" format from: http://arxiv.org/ps/hep-ph/9406364v1 
This figure "fig3-1.png" is available in "png" format from: http://arxiv.org/ps/hep-ph/9406364v1 
This figure "fig1-2.png" is available in "png" format from: http://arxiv.org/ps/hep-ph/9406364v1 
This figure "fig2-2.png" is available in "png" format from: http://arxiv.org/ps/hep-ph/9406364v1 
This figure "fig1-3.png" is available in "png" format from: http://arxiv.org/ps/hep-ph/9406364v1 
This figure "fig1-4.png" is available in "png" format from: http://arxiv.org/ps/hep-ph/9406364v1 
This figure "fig1-5.png" is available in "png" format from: http://arxiv.org/ps/hep-ph/9406364v1 
This figure "fig1-6.png" is available in "png" format from: http://arxiv.org/ps/hep-ph/9406364v1 